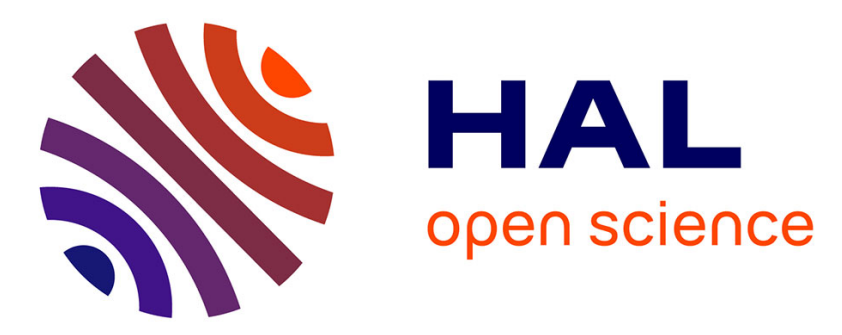

\title{
Analysis of the scattering of a plane acoustic wave by a doubly periodic structure using the finite element method: Application to Alberich anechoic coatings
}

Anne-Christine Hladky, Jean-noël Decarpigny

\section{- To cite this version:}

Anne-Christine Hladky, Jean-noël Decarpigny. Analysis of the scattering of a plane acoustic wave by a doubly periodic structure using the finite element method: Application to Alberich anechoic coatings. Journal of the Acoustical Society of America, 1991, 90 (6), pp.3356-3367. 10.1121/1.401395 . hal03300157

\section{HAL Id: hal-03300157 \\ https://hal.science/hal-03300157}

Submitted on 27 Jul 2021

HAL is a multi-disciplinary open access archive for the deposit and dissemination of scientific research documents, whether they are published or not. The documents may come from teaching and research institutions in France or abroad, or from public or private research centers.
L'archive ouverte pluridisciplinaire HAL, est destinée au dépôt et à la diffusion de documents scientifiques de niveau recherche, publiés ou non, émanant des établissements d'enseignement et de recherche français ou étrangers, des laboratoires publics ou privés. 


\title{
Analysis of the scattering of a plane acoustic wave by a doubly periodic structure using the finite element method: Application to Alberich anechoic coatings
}

\author{
Anne-Christine Hladky-Hennion and Jean-Noël Decarpigny \\ Acoustics Laboratory, ${ }^{\text {a) }}$ Institut Supérieur d'Electronique du Nord, 41 boulevard Vauban, \\ 59046 Lille Cedex, France
}

(Received 5 October 1990; accepted for publication 15 August 1991)

\begin{abstract}
The finite element approach has been previously used, with the help of the ATILA code, to model the scattering of acoustic waves by single periodic structures, such as compliant tube gratings [A. C. Hennion et al., J. Acoust. Soc. Am. 87, 1861-1870 (1990)]. In this paper, the same approach is extended to doubly periodic structures, such as Alberich anechoic coatings. To do this, only the unit cell of the periodic structure, including a small part of the surrounding fluid domain, has to be meshed, due to the use of classical Bloch type relations. Then, the effects of the remaining fluid domain are accounted for by matching the pressure field in the finite element mesh with simple plane-wave expansions of the ingoing and outgoing waves. After an outline of the method, the paper describes the results obtained for the scattering of a plane wave by different periodic structures. Internal losses are taken into account and the incident plane wave impinges at normal or oblique incidence. Numerical results obtained for Alberich anechoic coatings are first analyzed to check the convergence and then compared to previous numerical results or to experimental results, demonstrating that the finite element approach is accurate and well suited to predict the behavior of these gratings. Moreover, careful attention is devoted to the analysis of the inclusion vibrations, to identify the origin of the resonance mechanisms.
\end{abstract}

PACS numbers: 43.20.Fn, 43.20.Bi, 43.30.Gv, 43.30.Ky

\section{INTRODUCTION}

The scattering of a plane acoustic wave by a periodic array of elastic structures is widely used in underwater acoustics. In fact, such arrays can be efficient as reflecting screens or absorbers, within a large frequency band, and are used, for example, to increase the directivity and the acoustic level of low-frequency sources, to insulate receiving hydrophones from noise sources which are in proximity or to provide anechoic properties to the walls of acoustic tanks.

The immersed periodic structures can be split into two groups. The first one contains single periodic structures, such as single or double layered compliant tube gratings, which are directly immersed or embedded in a viscoelastic medium. In this case, and in the frequency band of interest, the incident plane wave excites a resonance mode of the tubes and the grating behaves as a refiecting baffle. ${ }^{1-9}$ The second group contains doubly periodic structures such as Alberich anechoic coatings. ${ }^{10-14}$ These coatings are multilayered structures in which one or several layers made up of absorbing materials contain doubly periodic inclusions, such as spherical or cylindrical cavities. Then, in the frequency band of interest, the incident wave excites a resonance mode of the inclusions and the coating behaves as a sound absorber. In order to explain the physical behavior and to help the design of such structures, several authors have built accurate mathematical models, which provide insertion loss values in nice agreement with measurements. On the one

\footnotetext{
.) Associated with the CNRS, U.R.A. 253.
}

hand, Burke et al, ${ }^{1}$ Dumery, ${ }^{2}$ Brigham et al., ${ }^{4}$ and Audoly et $a l^{8.9}$ have analyzed the behavior of gratings of circular cylinders as well as of arbitrarily oriented, elliptically shaped compliant tubes, using a multiple scattering approach. On the other hand, Vovk et al., ${ }^{3}$ Radlinski et al., ${ }^{5.7}$ and Audoly ${ }^{6}$ have used a waveguide approach to describe the behavior of gratings made up of tubes with elongated rectangular section. The study of spherical inclusions in an elastic layer has been performed by Gaunaurd, ${ }^{12,15,16}$ using homogeneous properties of the perforated solid. Finally, the diffraction of a plane wave impinging on a grating has been studied, using a boundary element method, by Achenbach et al. ${ }^{17}$ and Vidoret, ${ }^{18}$ while similar problems concerning the diffraction of elastic waves by a periodic array of cylinders have been solved using the T-matrix method by Lakhtakia et al..$^{19,20}$ These methods are powerful but require in most of the cases, a lot of specific algebraic developments as well as simplifying hypotheses for the displacement field, which restrict their use to a small number of given geometries. On the contrary, the efficiency and the versatility of the finite element approach to describe the acoustical behavior of single periodic structures ${ }^{21-23}$ has been demonstrated, particularly because this approach allows the modeling of any tube geometry, made up with any materials, by simply building the specific mesh. The aim of this article is to extend this finite element approach, previously described for single periodic structures, to doubly periodic structures, and to use it for analyzing the acoustical behavior of Alberich anechoic coatings.

Following this approach, a tridimensional mathematical model has to be used. The whole domain is split into 
three successive regions, separated by two planes that are parallel to the scattering structure plane. The first and third regions are semi-infinite fluid domains, in which the pressure field is expanded as a series of plane waves, either propagating or evanescent. In the first region, one of the propagating waves is the incident wave. The second region includes the scattering structures and a small part of the surrounding fluid domain. Using classical Bloch-type relations between displacement or pressure values at points that are separated by the grating spacing, only one unit cell of this second region has to be meshed with finite elements. ${ }^{21-24}$ Within this cell, a phase relation is applied on nodes separated by one period, defining boundary conditions between adjacent cells. Moreover, continuity equations for the pressure field and its normal derivative are written at the boundary between the mesh and the first and third regions, to take the effects of the external fluid domains into account. Following this method, the elastic structures are described by a finite element mesh, using the ATILA code which has been presented previous1y. ${ }^{25-29}$ Thus there is no restrictive hypothesis related to the displacement field, and modifying the structure only requires to modify the mesh, without any new development related to the method.

In the first part of this paper, the theoretical formulation is summed up, with emphasis on the aspects related to the periodicity. In the second part, one test case is first presented, which allows a validation of the method by comparison with previously obtained theoretical results. Then, after a careful discussion of the convergence with respect to the mesh step, Alberich coatings with periodic spherical or cylindrical inclusions are considered and the accuracy of the method for a plane wave at any incidence is demonstrated by comparison with measurements. Problems related to material parameters are discussed, and a careful analysis of the inclusion vibrations is performed, to identify the mechanical origin of the resonance effect. Finally, extensions of this approach to triply periodic structures or to active periodic structures are briefly discussed.

\section{THEORETICAL FORMULATION}

\section{A. General mathematical model for a doubly periodic structure}

For the presentation of the model, although a lot of other cases can also be dealt with, the doubly periodic structure of reference is an Alberich anechoic layer, the cross section and top views of which are described on Fig. 1. It is a multilayered structure, made up of a viscoelastic material, containing air inclusions. This structure is immersed and its plane is set normal to the $z$ axis. It is theoretically assumed to be infinite in the $x$ and $y$ directions, and to be doubly periodic. Thus the problem is tridimensional. The whole domain is split into three successive regions, by two planes that are parallel to the grating plane. These regions have the same characteristics as in the case of previously analyzed single periodic structures. ${ }^{22}$ The first and the third regions are semi-infinite fluid domains. The second region, which includes the scattering structure and its immediate surrounding fluid domain is detailed in Fig. 2. The $S_{+}$and $S_{-}$surfaces, parallel to the $x 0 y$ plane, limit the finite element mesh
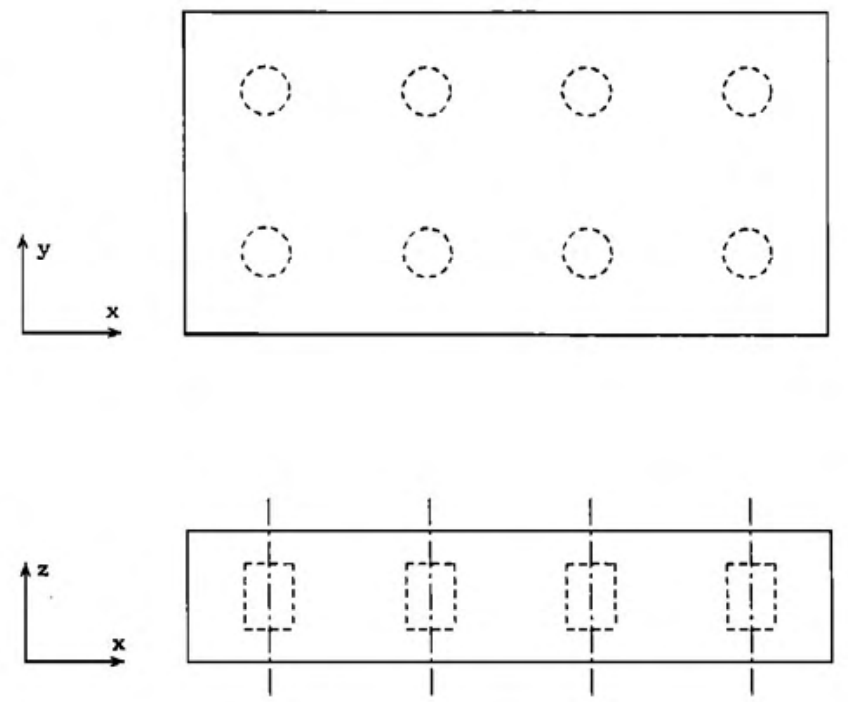

FIG. 1. Cross-section and top views of the reference Alberich anechoic layer.

and represent, on the one hand, the boundary between regions I and II and, on the other hand, the boundary between regions II and III. Moreover, due to the periodicity of the grating, the $S 1$ and $S 3$ planes, parallel to the $y 0 z$ plane, and the $S 2$ and $S 4$ planes, parallel to the $x 0 z$ plane, limit the unit cell, which is $2 d_{1}$ wide in the $x$ direction and $2 d_{2}$ wide in the $y$ direction. Then, the grating is excited by a plane, monochromatic wave, the direction of incidence of which is marked by two angles $\theta$ and $\varphi$ (Fig. 2). The time dependence being written as $e^{-j \omega t}$, the incident wave expression is

$$
\begin{aligned}
p_{i}(t, x, y, z)= & p_{i} \exp [j k(x \sin \theta \cos \varphi \\
& +y \sin \theta \sin \varphi+z \cos \theta)] e^{-j \omega t} \\
= & e^{-j \omega t} p_{i}(x, y, z),
\end{aligned}
$$

where $k$ and $\omega$ are, respectively, the wave number and the angular frequency in the fluid.

Because the grating is assumed to spread from $-\infty$ to $+\infty$ in the $x$ and $y$ directions and to be periodic, any space function $F$ (pressure, displacement...) has to verify the relation

$$
\begin{aligned}
& F\left(x+2 d_{1}, y+2 d_{2}, z\right) \\
& \quad=F(x, y, z) e^{2 d d_{1} k \sin \theta \cos \varphi} e^{2 d_{2} k \sin \theta \sin \varphi} .
\end{aligned}
$$

Using this relation, the solution of the time-independent wave equation for region I can be written in the following form:

$p^{-}(x, y, z)=p_{i}(x, y, z)$

$$
+\sum_{n, m=-\infty}^{+\infty} R_{n m}^{p} e^{-j k_{n m^{2}} e^{j \alpha_{n} x} e^{j \beta_{m} y}}
$$

where

$$
\begin{aligned}
& \alpha_{n}=n \pi / d_{1}+k \sin \theta \cos \varphi, \\
& \beta_{m}=m \pi / d_{2}+k \sin \theta \sin \varphi, \\
& k_{n m}^{2}=k^{2}-\alpha_{n}^{2}-\beta_{m}^{2} .
\end{aligned}
$$

The incident wave is given by the first term. The second term represents a doubly infinite series of waves reflected by the 


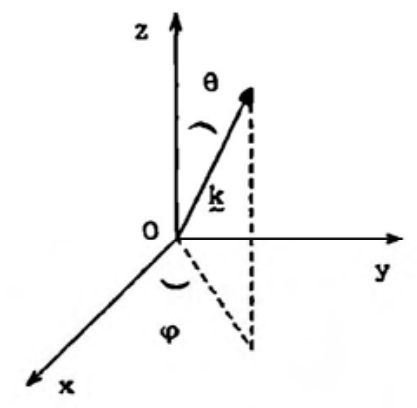

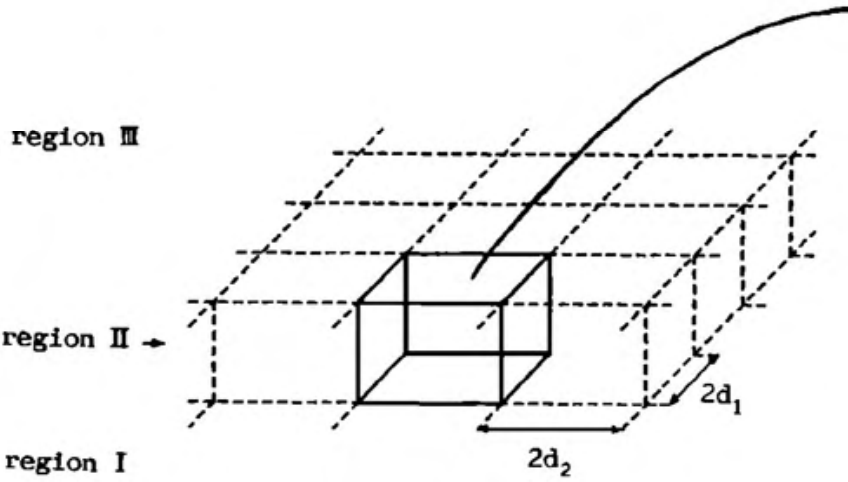

region I $\mathrm{s}$.

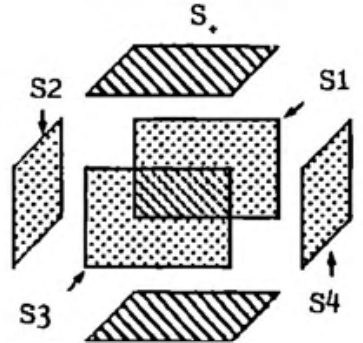

S.
FIG. 2. Schematic description of a doubly periodic structure, used to define $S_{-}, S_{+}, S 1, S 2, S 3$, and $S 4$ planes and definition of the incidence angles: $\mathbf{k}$ is the incident wave vector. grating, the $R_{n m}^{p}$ terms being reflection coefficients to be determined. When $k_{n m}^{2}<0$, the corresponding wave propagates parallel to the $x 0 y$ plane and decays exponentially in the negative $z$ direction, away from the grating. It is referred to as an evanescent wave. On the other hand, if $k_{n m}^{2}>0$, the wave propagates away from the grating in the negative $z$ direction. In this case, its propagating direction is determined by the wave vector $\left(\alpha_{n}, \beta_{m},-k_{n m}\right)$. So, interferences have to take place when more than one propagating wave exists and disturb reflection measurements. In the same way, the transmitted pressure in region III is given by

$$
p^{+}(x, y, z)=\sum_{n, m=-\infty}^{+\infty} T_{n m}^{p} e^{j k_{n m^{2}}} e^{j \alpha_{n} x} e^{\beta_{m} y},
$$

where the $T_{n m}^{p}$ terms are transmission coefficients to be determined. A particular interest in this problem is to calculate the transmitted and reflected pressures far from the grating. If the following condition is verified,

$\omega<\min \left(\frac{c \pi}{d_{1}(1+|\sin \theta \cos \varphi|)}, \frac{c \pi}{d_{2}(1+|\sin \theta \sin \varphi|)}\right)$,

only one propagating wave exists and the transmitted and reflected pressures in the far field are determined, respectively, by $T_{o 0}^{p}$ and $R_{o 0}^{p}$. All other waves are evanescent and do not contribute to this far field.

In the same way, the pressure normal gradient can be expanded as a series of propagating and evanescent waves.

\section{B. Finite element modeling of the unit cell}

Due to the use of the classical Bloch type relation (2) between the displacement or pressure values at points which are separated by a grating spacing, only one unit cell of re- gion II is meshed using finite elements. ${ }^{23.30}$ Writing relation (2) provides the boundary conditions between adjacent cells and writing continuity equations for the pressure field and its normal derivative provides the boundary conditions on surfaces $S_{+}$and $S_{-}$. These points are discussed in details in Refs. 22 and 23.

Then, using the finite element method, with the help of the ATILA code, ${ }^{22,23,26-29}$ the unit cell is meshed and divided into elements connected by nodes. A cross section of this unit cell in the $x 0 z$ plane is shown in Fig. 3: The solid structure $\Omega_{s}$ is in contact with the infinite fluid domain $\Omega_{f}$,

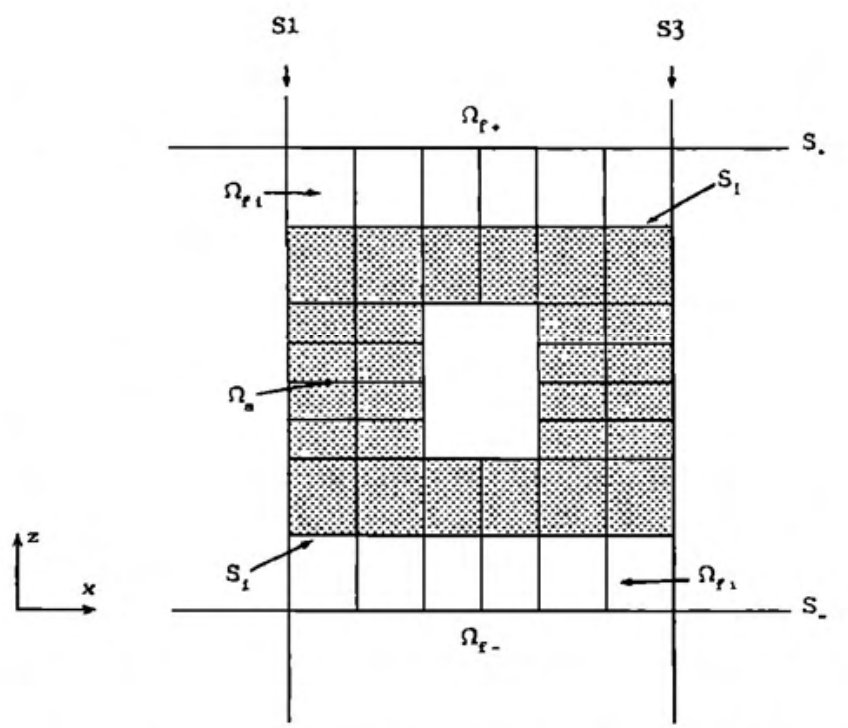

FIG. 3. Finite element mesh of the cross section of the unit cell, in the $x 0 z$ plane, used to define $\Omega_{s}, \Omega_{f}, \Omega_{f-}$, and $\Omega_{f+}$ subdomains. 
ture $\Omega_{s}$ is in contact with the infinite fluid domain $\Omega_{f}$, through the surface $S_{i} . \Omega_{f}$ is split into three parts, $\Omega_{f}, \Omega_{f+}$, and $\Omega_{f-}$, by the surfaces $S_{+}$and $S_{-}$. If a steady-state harmonic analysis is considered, the whole system of equations is, classically,

$$
\left(\begin{array}{cc}
{[K]-\omega^{2}[M]} & -[L] \\
-\rho^{2} c^{2} \omega^{2}[L]^{T} & {[H]-\omega^{2}\left[M_{1}\right]}
\end{array}\right)\left(\begin{array}{l}
\mathbf{U} \\
\mathbf{P}
\end{array}\right)=\left(\begin{array}{c}
\mathbf{F} \\
\rho c^{2} \Phi
\end{array}\right),
$$

where the unknowns are the vectors of nodal values of the pressure $\mathbf{P}$ and of the displacement $\mathbf{U}$. $[K]$ and $[M]$ are, respectively, the structure stiffness and coherent mass matrices, while $[H]$ and $\left[M_{1}\right]$ are the fluid compressibility and coherent mass matrices. $[L]$ is a connectivity matrix that corresponds to the coupling between the structure and the fluid and is related to the kinematical and dynamical interface continuity equations. ${ }^{25,27} \rho$ and $c$ are, respectively, the fluid density and sound speed, $\omega$ is the angular frequency. $\mathbf{F}$ contains the nodal values of the applied forces, and $\Phi$ contains the nodal values of the pressure normal gradient on the fluid domain boundaries $S_{+}$and $S_{-} \cdot[K],[M],[H]$, $\left[M_{1}\right],[L], \mathbf{F}$, and $\Phi$ result, respectively, from the merging of elementary matrices and vectors $\left[K^{e}\right],\left[M^{e}\right],\left[H^{e}\right]$, $\left[M_{1}^{e}\right],\left[L^{e}\right], \mathbf{F}^{e}$, and $\Phi^{e}$, where $e$ stands for the element number. This system can take into account damping in the materials via the use of complex elastic constants, ${ }^{22,29}$ depending or not upon the frequency. In all the following examples, isoparametric elements are used, with a quadratic interpolation along element sides.

\section{Specific modifications of the finite element equations}

The application of the periodic boundary conditions implies that the phase relation (2) between nodal values belonging to the $S 1$ and $S 3$ planes, on the one hand, to the $S 2$ and $S 4$ planes, on the other hand, has to be incorporated in the matrix equation (7). ${ }^{22,23,30}$ The corresponding relations are detailed in Appendix A. The resulting matrix is complex but Hermitean. In terms of the finite element method, this operation is a static condensation of the degrees of freedom belonging to $S 3$ and $S 4$.

To take into account the effects of the remaining fluid domain, above the $S_{+}$surface and below the $S_{-}$surface, the pressure field described by the finite element interpolation is matched with the plane-wave expansions of the ingoing and outgoing waves, given by Eqs. (3) and (5). These series are restricted to $\left(2 M_{x}+1\right) \cdot\left(2 M_{y}+1\right)$ terms, where $M_{x}$ and $M_{y}$ are parameters which depend upon the mesh steps on the $S_{+}$and $S_{-}$surfaces, in the $x$ and $y$ directions, respectively. The formalism has already been described ${ }^{22,23}$ in the case of single periodic structures and the equations are detailed in Appendix B. Writing these continuity equations introduces matrix relations between the nodal values of the reflected pressure normal derivative on the $S_{-}$surface (respectively transmitted pressure normal derivative on the $S_{+}$ surface) and the nodal values of the reflected pressure on the $S_{-}$surface (respectively transmitted pressure on the $S_{+}$ surface):

$$
\Phi_{+}=\left[\Delta^{+}\right] \mathbf{P}_{+}, \quad \Phi_{-}=2 \Phi_{i}+\left[\Delta^{-}\right] \mathbf{P}_{-},
$$

where vector $\Phi_{i}$ contains the nodal values of the incident pressure normal gradient. Full expressions of matrices $\left[\Delta^{+}\right]$and $\left[\Delta^{-}\right]$are given by the relations (B12) and (B13). These matrices can then be incorporated in system (7) and, finally, the whole matrix equation is

$$
\begin{gathered}
\left(\begin{array}{cc}
{\left[K^{\prime}\right]-\omega^{2}\left[M^{\prime}\right]} & -\left[L^{\prime}\right] \\
-\rho^{2} c^{2} \omega^{2}\left[L^{\prime}\right]^{T^{*}} & {\left[H^{\prime}\right]-\omega^{2}\left[M_{1}^{\prime}\right]-\rho c^{2}[\Delta]}
\end{array}\right)\left(\begin{array}{l}
\mathbf{U} \\
\mathbf{P}
\end{array}\right) \\
=\left(\begin{array}{c}
0 \\
\rho c^{2} \Phi^{\prime}
\end{array}\right) .
\end{gathered}
$$

In this matrix equation, the vectors $\mathbf{P}$ and $\Phi^{\prime}$, as well as the $[\Delta]$ matrix, are partitioned with respect to three domains which are the $S_{+}$surface, the $S_{-}$surface and the inner fluid domain $\Omega_{f}$. They can be written under the form

$$
\begin{gathered}
\mathbf{P}=\left(\begin{array}{c}
\mathbf{P}_{+} \\
\mathbf{P}_{-} \\
\mathbf{P}_{f i}
\end{array}\right), \quad \Phi^{\prime}=\left(\begin{array}{c}
\mathbf{0} \\
2 \Phi_{i}^{\prime} \\
\mathbf{0}
\end{array}\right), \\
{[\Delta]=\left(\begin{array}{ccc}
{\left[\Delta^{+}\right]} & {[0]} & {[0]} \\
{[0]} & {\left[\Delta^{-}\right]} & {[0]} \\
{[0]} & {[0]} & {[0]}
\end{array}\right) .}
\end{gathered}
$$

The prime indicates that each term has been submitted to the static condensation of the solid as well as fluid degrees of freedom between the nodes of the $(S 1)$ and $(S 3)$ surfaces, on the one hand, of the $(S 2)$ and $(S 4)$ surfaces on the other hand.

\section{RESULTS}

Using the finite element method described above, frequency variations of the transmission coefficient have been investigated for different types of gratings. The transmission coefficient, as used here, is given by

$$
T=20 \log \left|P^{\mathrm{tr}} / P^{i}\right|,
$$

where $P^{\text {tr }}$ is the transmitted pressure amplitude, computed with the help of the $T_{00}^{p}$ term of the expression (5) and $P^{i}$ is the incident pressure amplitude. For the configurations that are modeled hereafter, only one propagating wave is generally considered, with the same direction as the incident wave, the other waves being assumed to be evanescent. In all the cases, even if other waves propagate in the two semi-infinite fluid spaces, the transmission coefficient is calculated by using the $T_{00}^{p}$ term of expression (5). In each example, for the Young's modulus as well as for the Poisson's ratio, the loss angle is defined as the absolute value of the ratio of the imaginary part to the real part and given as a percentage.

In the case of plates containing spherical or cylindrical inclusions, for which transmission coefficient measurements have been performed, the material can be either polyurethane or silicone. In these cases, the physical properties depend upon the experimental temperature and the frequency. They have been read from master curves, provided by the manufacturer, and updated parameter values have been used for the computation at each frequency. 


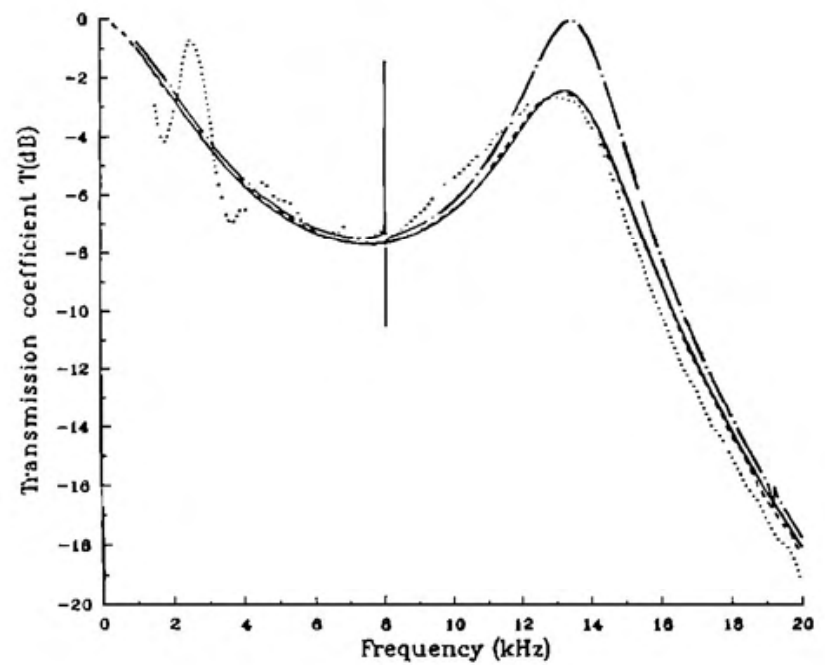

FIG. 4. Frequency variations of the transmission coefficient for a plastic circular cylinder grating: dashed dotted line: FEM $0 \%$ loss; full line: FEM $6 \%$ loss; dashed line: analytical method $6 \%$ loss; dotted line: measurements.

\section{A. Transmission coefficient for plastic circular cylinder gratings}

In this section, a single periodic structure has been modeled using a tridimensional mesh, to validate the method. The test example is a plastic tube grating, the tubes being made of PVC the physical constants of which are $\rho=1400$ $\mathrm{kg} / \mathrm{m}^{3}, E=4.1 \times 10^{9} \mathrm{~Pa}, v=0.4$. The tube external radius is $2.5 \mathrm{~cm}$. The thickness of the wall is $3 \mathrm{~mm}$. The grating spacing is $6 \mathrm{~cm}$. The loss angle for the PVC Young's modulus is chosen equal to $6 \%$. This structure has first been modeled using a bidimensional mesh ${ }^{22}$ and the finite element results, taking account or not of losses in the PVC, have been compared with measurements and with results from a previous analytical model. ${ }^{2,9}$ A good agreement has been obtained between the different approaches, as shown by Fig. 4. Below $8 \mathrm{kHz}$, the diffraction effects on the experimental curve are due to the finite size of the panel. Then, a tridimensional elementary cell has been built (Fig. 5), by cutting a segment of the tube, but to save the validity of the plane strain hypothesis assumed in the bidimensional case, the length of the segment has been retained small compared to the wavelength. Results obtained in this three-dimensional case, for the same incident wave, are strictly identical to those obtained in the previous bidimensional case, and thus are not reproduced. Excluding losses in the PVC, the real and the imaginary parts of the displacement field at $13.5 \mathrm{kHz}$ are displayed on Fig. 6, demonstrating that the plane strain hypothesis is verified, generating lines of the cylinder remaining strictly parallel.

\section{B. Convergence and mesh step selection}

The Alberich anechoic coatings, which are studied in Secs. II C and II D, are made of polyurethane or silicone. In the frequency range of interest, the transverse wave velocity $c_{t}$ in these materials is very low (between 200 and $300 \mathrm{~m} / \mathrm{s}$ for the polyurethane, about $20 \mathrm{~m} / \mathrm{s}$ for the silicone), while the longitudinal wave velocity $c_{1}$ is close to $1700 \mathrm{~m} / \mathrm{s}$.<smiles>CC(C)C</smiles>

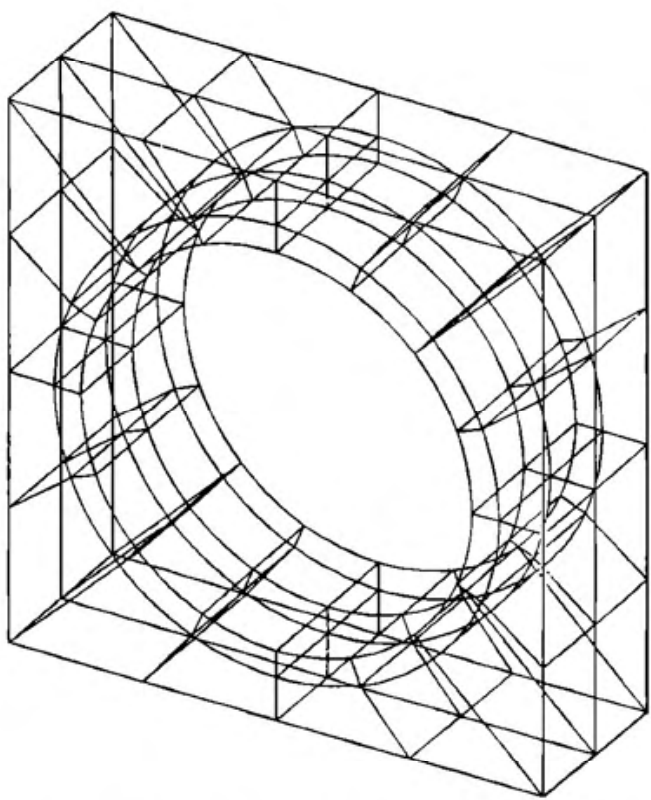

FIG. 5. Three-dimensional finite element mesh for the elementary cell of the plastic circular cylinder grating.

Moreover, the finite element formulation used here relying upon quadratic interpolation functions, the classical $\lambda / 4 \mathrm{cri}-$ terion, ${ }^{31}$ which states that the largest length of each element in a given mesh has to be smaller than a quarter of the transverse wavelength in the material for the working frequency has to be verified. Thus, due to the small $c_{\text {t }}$ values, meshes have to be very tight. To test the validity of the $\lambda / 4$ rule in the present cases and to determine the sensitivity of the result accuracy on the checking of this rule, two specific examples have been studied that allow one to work with several different meshes at reasonable computer costs.

First, a single periodic structure has been analyzed. A polyurethane panel is considered, the properties of which are assumed to be constant in all the frequency range, corresponding to $5^{\circ} \mathrm{C}$ and $16 \mathrm{kHz}$ in Table $\mathrm{I}$. This panel is $4 \mathrm{~cm}$ thick and contains infinite air tubes arranged as a grating whose spacing is $5 \mathrm{~cm}$. The tube cross sections are rectangles that are $2 \mathrm{~cm}$ high and $1.5 \mathrm{~cm}$ large. The model is a bidimensional mesh, assuming plane strain conditions. Five meshes have been considered. At $15 \mathrm{kHz}$, which is close to the resonance frequency, the first mesh verifies a $\lambda / 2$ criterion for $c_{t}$, the second a $\lambda / 4$ criterion, the third a $\lambda / 6$ criterion, the fourth a $\lambda / 8$ criterion, and, finally, the fifth a $\lambda / 12$ criterion. Figure 7 displays the variations of the panel transmission coefficient versus frequency, when the excitation is a normal incident plane wave. Curves demonstrate that using a $\lambda / 12$, $\lambda / 8, \lambda / 6$, or $\lambda / 4$ criterion with respect to $c_{t}$ gives results in very close agreement. The resonance frequencies remain quite identical, the relative differences being less than $1.3 \%$, while the minimum level variations are smaller than $1 \mathrm{~dB}$. Moreover, $\mathrm{a} \lambda / 2$ mesh step is clearly insufficient to describe 
$\stackrel{n}{*}$
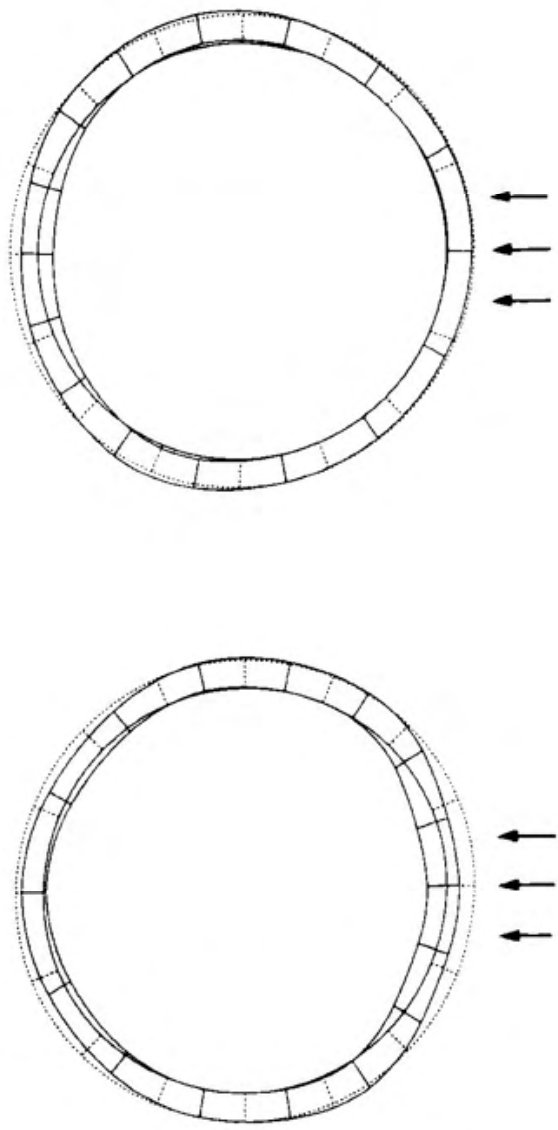

$y_{n}^{x}$
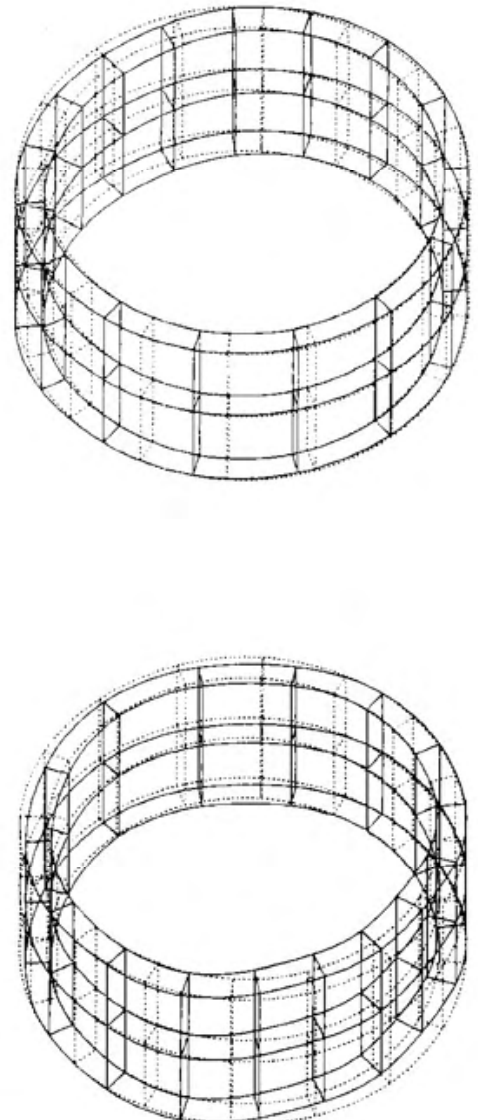

FIG. 6. Real part and imaginary part of the displacement field for the segment of cylinder, without losses, at $13.5 \mathrm{kHz}$, (a) in the $x 0 z$ plane and (b) in $3-\mathrm{D}$ view. Dashed lines correspond to the rest position and the displacement amplitude is arbitrary.

perfectly the resonance, but still provide a reasonable sketch. These results are obtained while the displacement field associated to the resonance frequency and displayed in Fig. 7 is very similar to the displacement fields obtained for actual Alberich structures.

Second, an Alberich anechoic coating has been modeled, which grating spacing is sufficiently small to allow low computation times. The panel is $2 \mathrm{~cm}$ thick. The cylin-

TABLE I. Young's modulus $E$ (in $10^{8} \mathrm{~Pa}$ ), Poisson's ratio $v$ and loss factors (in \%) for the Young's modulus ( $\left.\operatorname{tg} \delta_{E}\right)$ and the Poisson's ratio $\left(\operatorname{tg} \delta_{v}\right)$, versus the frequency (in $\mathrm{kHz}$ ) and the temperature $\left(\right.$ in ${ }^{\circ} \mathrm{C}$ ) for the polyurethane.

\begin{tabular}{|c|c|c|c|c|c|c|c|c|}
\hline \multirow[b]{2}{*}{$f$} & \multicolumn{4}{|c|}{$T=5^{\circ} \mathrm{C}$} & \multicolumn{4}{|c|}{$T=20^{\circ} \mathrm{C}$} \\
\hline & $E$ & $\operatorname{tg} \delta_{E}$ & $v$ & $\operatorname{tg} \delta_{v}$ & $E$ & $\operatorname{tg} \delta_{E}$ & $v$ & $\operatorname{tg} \delta_{v}$ \\
\hline 2 & 1.53 & 45.0 & 0.489 & 0.87 & 0.71 & 37.8 & 0.494 & 0.38 \\
\hline 4 & 1.88 & 45.0 & 0.486 & 1.08 & 0.84 & 40.2 & 0.493 & 0.50 \\
\hline 6 & 2.11 & 45.0 & 0.485 & 1.24 & 0.94 & 41.7 & 0.492 & 0.58 \\
\hline 8 & 2.30 & 45.0 & 0.483 & 1.36 & 1.01 & 42.9 & 0.492 & 0.64 \\
\hline 10 & 2.45 & 45.0 & 0.482 & 1.46 & 1.06 & 43.8 & 0.491 & 0.70 \\
\hline 12 & 2.59 & 45.0 & 0.481 & 1.55 & 1.11 & 44.5 & 0.491 & 0.74 \\
\hline 14 & 2.70 & 45.0 & 0.480 & 1.64 & 1.16 & 45.1 & 0.490 & 0.79 \\
\hline 16 & 2.81 & 45.0 & 0.479 & 1.78 & 1.20 & 45.7 & 0.490 & 0.83 \\
\hline 18 & 2.91 & 45.0 & 0.478 & 1.81 & 1.23 & 46.1 & 0.490 & 0.86 \\
\hline 20 & 3.00 & 45.0 & 0.478 & 1.85 & 1.27 & 46.6 & 0.489 & 0.90 \\
\hline
\end{tabular}

drical inclusions are $1.5 \mathrm{~cm}$ high and their diameter is equal to $2 \mathrm{~cm}$. These inclusions are arranged as a doubly periodic structure, whose grating spacing is $3 \mathrm{~cm}$. The properties of the panel material are $E=1.4 \times 10^{9} \mathrm{~Pa}, \rho=1100 \mathrm{~kg} / \mathrm{m}^{3}$, $v=0.49$, the loss factor for the Young's modulus being equal to $23 \%$. Figure 8 presents the top views of three different meshes of the unit cell. At $3 \mathrm{kHz}$, mesh 8 (a) verifies a $\lambda / 2$ criterion with respect to $c_{t}$, mesh 8 (b) a $\lambda / 4$ criterion, and mesh 8 (c) a $\lambda / 12$ criterion. Figure 9 presents the variations of the panel transmission coefficient versus frequency, when it is excited by a plane wave at normal incidence. It shows that, in the frequency range of interest, the three curves have identical shapes. But, at the resonance frequency, the convergence is not reached and a shift of several hundred Hertz is observed between successive meshes. In fact, at $3 \mathrm{kHz}$, and as demonstrated by Fig. 9, the resonance is mainly due to a flexural motion of the cover layer. Thus, the $\lambda / 4$ criterion must be written using the flexural wave velocity which is, in this case, about $70 \mathrm{~m} / \mathrm{s}^{32}$ Thus, the actual $\lambda / 4$ criterion corresponds roughly to $\lambda / 12$ criterion for $c_{l}$, which explains the frequency shift.

These results demonstrate that, if the transverse and longitudinal wave velocities are the relevant parameters, the checking of a $\lambda / 4$ criterion with respect to $c$ is highly sufficient. Nevertheless, in some cases, if for example cavity cover layers are too thin, the relevant velocity can be the 

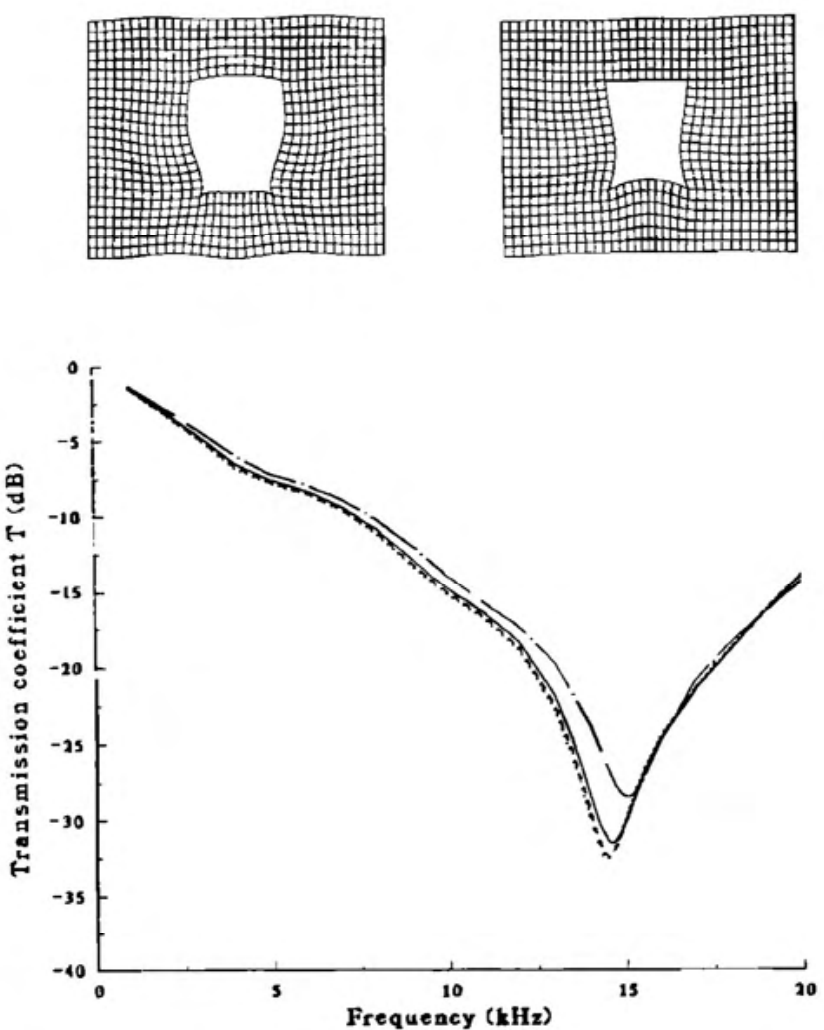

FIG. 7. Frequency variations of the transmission coefficient of the panel containing air rectangular tubes (Sec. II B). Dashed dotted line: the mesh verifies the $\lambda / 2$ criterion for $c_{1}$ at $15 \mathrm{kHz}$; full line: the mesh verifies the $\lambda / 4$ criterion; dashed line: the mesh verifies the $\lambda / 6$ criterion; dotted line: the mesh verifies the $\lambda / 8$ or $\lambda / 12$ criterion (same curve). The inserted graphic displays are the real and imaginary parts of the displacement field for an elementary cell of the panel cross section, at $14.4 \mathrm{kHz}$, for the tightest mesh.

flexural wave velocity $c_{f}$, which is frequency dependent, and in this case tighter meshes can be useful. Thus, if this situation seems to be possible, $c_{t}$ and $c_{f}$ must be compared before selecting the appropriate mesh step.

\section{Transmission coefficient from coatings with periodic spherical inclusions}

In this section, a plate containing periodic spherical air inclusions is modeled, with a view to analyzing the inclusion vibrations with the help of the computed displacement fields. The panel, made of polyurethane, is $5 \mathrm{~cm}$ thick and
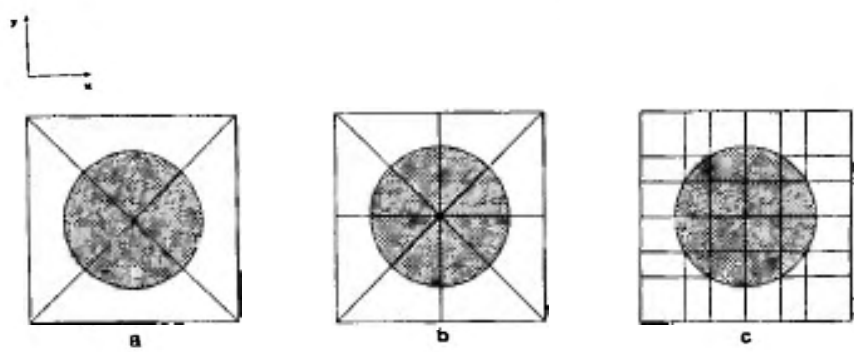

FIG. 8. Top views of the finite element meshes of the elementary cell for the Alberich anechoic coating studied in Sec. II B. The dotted domain is the air cavity. At $3 \mathrm{kHz}$, mesh (a) verifies a $\lambda / 2$ criterion for $c_{t}$, mesh (b) a $\lambda / 4$ criterion, mesh (c) a $\lambda / 12$ criterion.
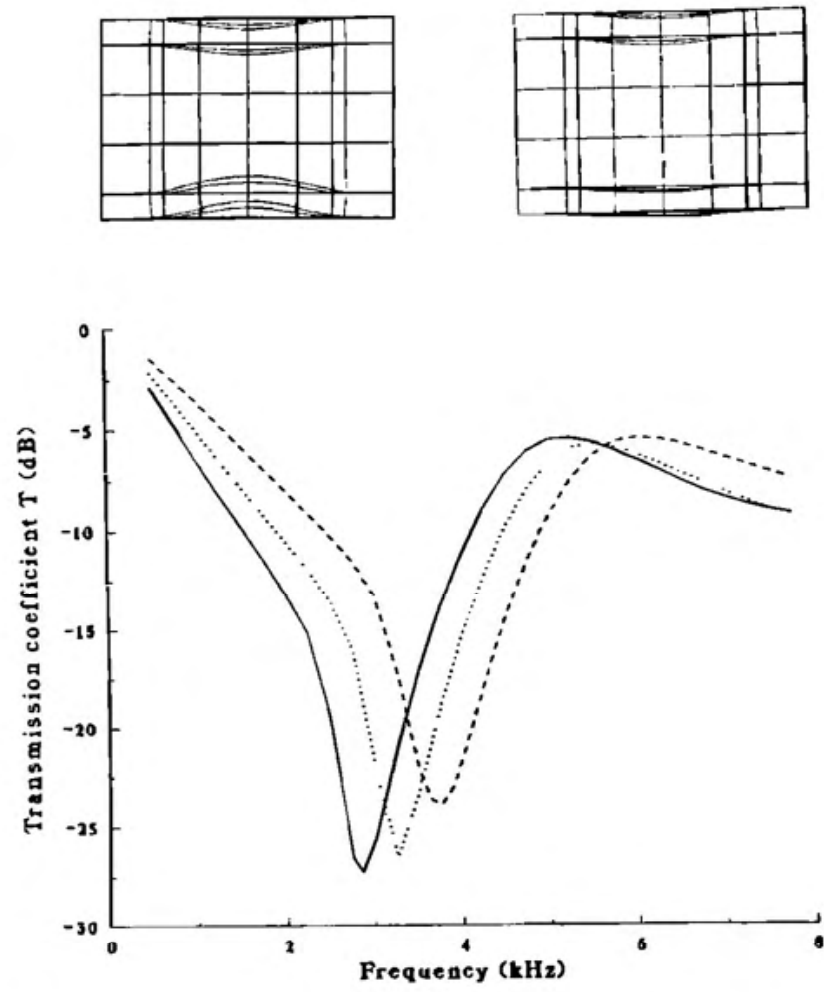

FIG. 9. Frequency variations of the transmission coefficient of the Alberich anechoic coating studied in Sec. II B: dashed line: FEM mesh 8(a); dotted line: FEM mesh 8(b); full line: FEM mesh 8(c). The inserted graphic displays are the real and imaginary parts of the displacement field of the panel, at $2.85 \mathrm{kHz}$, in the $x 0 z$ plane, for the tightest mesh.

contains air bubbles, the radius of which is $1 \mathrm{~cm}$. They form a doubly periodic structure, the grating spacing of which is 5 $\mathrm{cm}$. The density of the polyurethane is $1100 \mathrm{~kg} / \mathrm{m}^{3}$ and the other properties, which depend upon frequency and temperature, are given in Table $I$. Then, the panel is immersed in water, where the density and sound speed are assumed to be $1000 \mathrm{~kg} / \mathrm{m}^{3}$ and $1489 \mathrm{~m} / \mathrm{s}$. The polyurethane temperature is equal to $20^{\circ} \mathrm{C}$. The $\lambda / 4$ criterion with respect to $c_{t}$ is sufficient and has been used to define the mesh, ensuring the convergence as discussed in Sec. II B. Figure 10 compares finite element results to measurements, when the grating is excited by a plane wave at normal incidence. The agreement is good between the two curves. The displacement field of the elementary cell is presented at $12 \mathrm{kHz}$, in the $x 0 z$ plane on Fig. 11, at eight different times, separated by one-eighth of the period, to describe the amplitude and phase field variations simultaneously inside the plate thickness. The amplitudes are normalized to the maximum displacement amplitude. The minimum of the transmission coefficient is due to the excitation of a resonance mode of the cavity but the cycle does not correspond to a pure radial mode. In fact, the internal losses in the polyurethane introduce an important phase shift in the displacement field. Nevertheless, the combination of the radial mode of the inclusion and of a translational vibration is predominant.

Then, to assess the effects of the experimental temperature on the curves, computations have been performed where the coating temperature is equal to $5{ }^{\circ} \mathrm{C}, 10^{\circ} \mathrm{C}, 15^{\circ} \mathrm{C}$, and $20^{\circ} \mathrm{C}$. In these cases, the properties of the polyurethane 


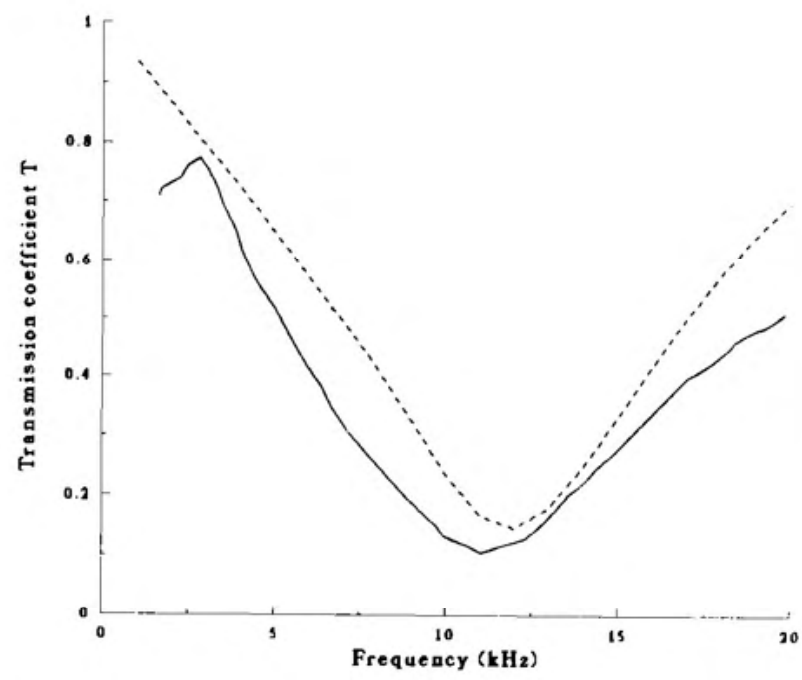

FIG. 10. Frequency variations of the transmission coefficient of the panel made of polyurethane, containing air spherical inclusions: full line: measurements; dashed line: FEM.
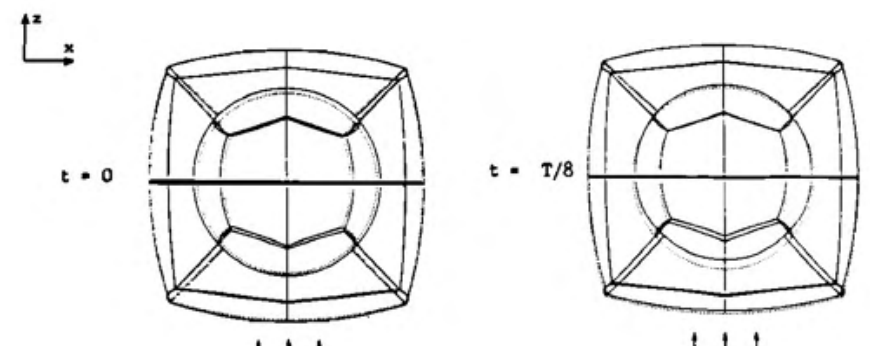

111

111

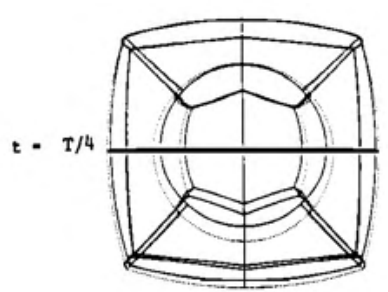

111

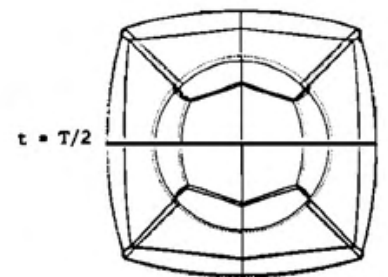

111

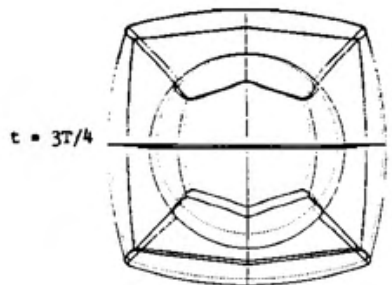

111

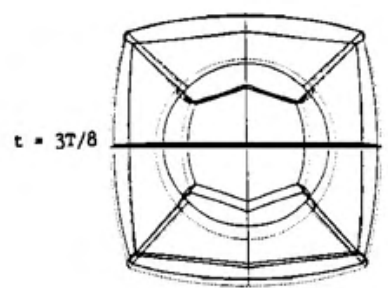

111

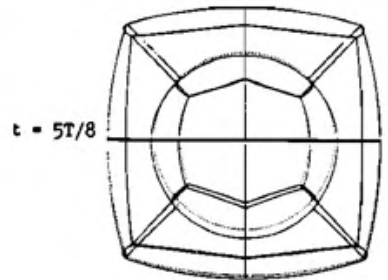

111

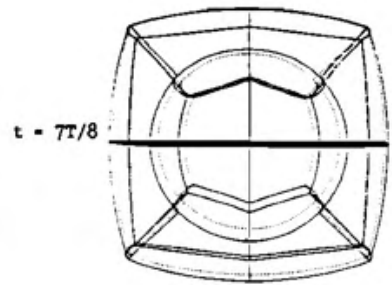

111
FIG. 11. Displacement field of one spherical inclusion inside the elementary cell of the panel made of polyurethane, at $12 \mathrm{kHz}$, in the $x 0 \mathrm{z}$ plane, at eight different moments, separated by one-eighth of the period (for sake of simplicity, only elements beside the cavity are displayed).

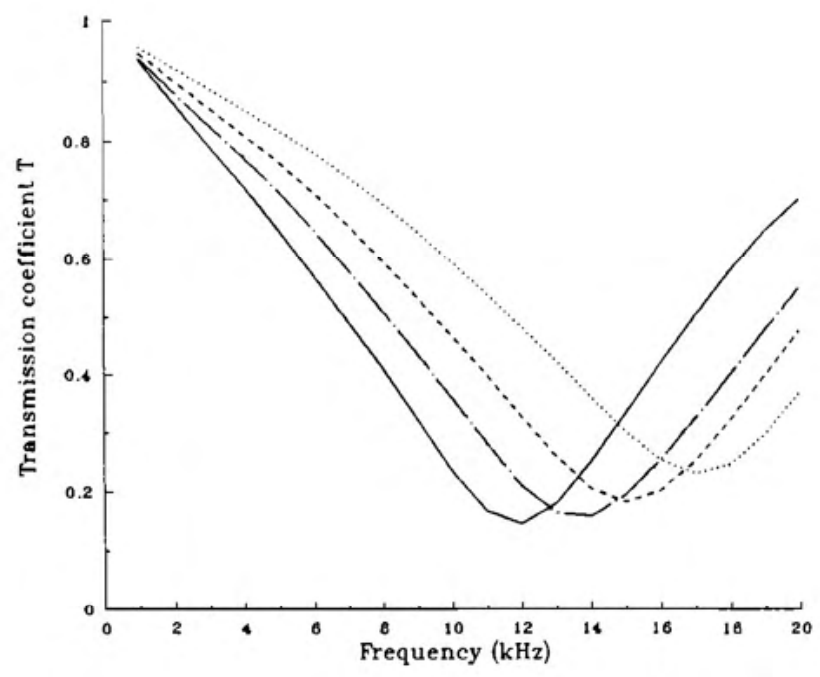

FIG. 12. Frequency variations of the transmission coefficient of the panel made of polyurethane, containing air spherical inclusions: full line: FEM $20^{\circ} \mathrm{C}$; dashed dotted line: FEM $15^{\circ} \mathrm{C}$; dashed line: FEM $10^{\circ} \mathrm{C}$; dotted line: FEM $5{ }^{\circ} \mathrm{C}$.

are changed at each frequency, following the master curves provided by the manufacturer. Figure 12 presents the finite element results and shows that temperature variations can strongly modify the position of the maximum of transmission losses as well as the bandwidth performance.

\section{Transmission coefficient from coatings with periodic cylindrical inclusions}

The Alberich anechoic coating described in Fig. 1 has been modeled and its acoustical behavior is analyzed in this section. The panel is $4 \mathrm{~cm}$ thick. The cylindrical inclusions, which are $2 \mathrm{~cm}$ high and whose diameter is $1.5 \mathrm{~cm}$, are arranged as a doubly periodic structure, whose grating spacing is $5 \mathrm{~cm}$.

\section{Alberich anechoic coating made of polyurethane}

The first panel considered is made of polyurethane, whose properties are given in Table I and assumed to be at $5^{\circ} \mathrm{C}$. In the frequency range of interest, the transverse wave velocity is the relevant parameter, the cavity cover layers being thick. The mesh used in this analysis is described on Fig. 13. It verifies the $\lambda / 4$ criterion with respect to $c_{t}$ for a frequency lower than $17 \mathrm{kHz}$. Figure 14 compares the finite element results with the measurements, when the grating is excited by a plane wave at normal incidence. The overall agreement is good and the shape of the curve is correct. However, a 2-kHz upward shift of the minimum transmission frequency is observed which has to be explained. In fact, conclusions that have been clearly demonstrated in Sec. II B preclude a convergence problem, a statement which is still reinforced by the discrepancy between the two curves even at frequencies lower than $10 \mathrm{kHz}$ where the mesh step is much smaller than $\lambda / 4$. Moreover, the kind of discrepancy on the lower side of the resonance is not observable in Fig. 7, even for a $\lambda / 2$ mesh. Thus the discrepancy is probably induced by the use of inaccurate values of polyurethane parameters. In fact, in the frequency and temperature ranges of interest, this 

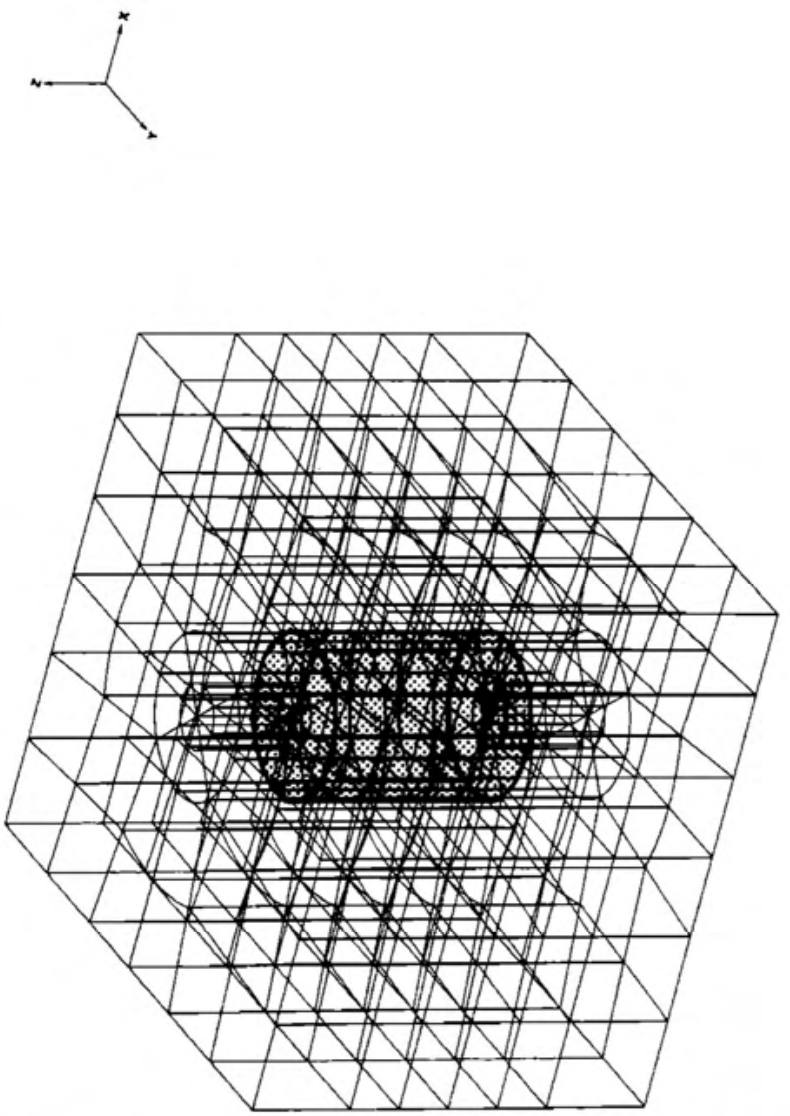

FIG. 13. Finite element mesh of the elementary cell for the Alberich anechoic coating. The dotted domain is the air cavity (air is not modeled).

material exhibits a transition and elastic coefficients can undergo large variations. Thus new computations have been performed with a $25 \%$ reduced Young's modulus for the polyurethane at each frequency. Figure 14 displays the corresponding finite element results. This time, the calculated curve is in much better agreement with the measurements, demonstrating that the previous discrepancy can be definite-

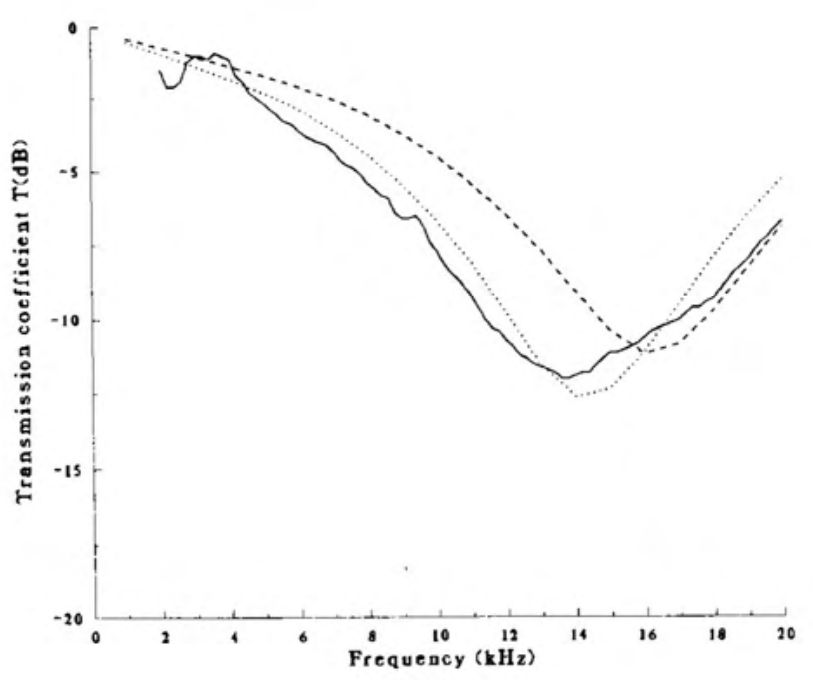

FIG. 14. Frequency variations of the transmission coefficient of the Alberich anechoic coating, made of polyurethane: full line: measurements; dashed line: FEM; dotted line: FEM with adjusted properties.

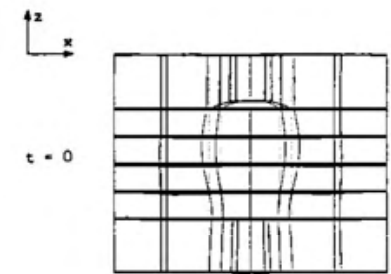

111

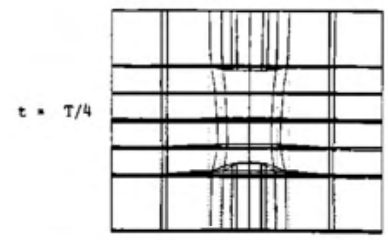

111

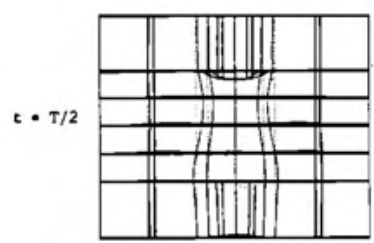

111

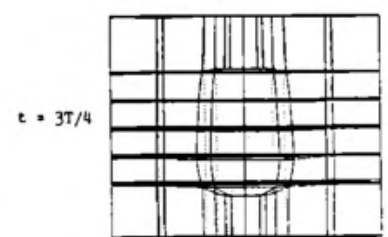

$1 i 1$
111

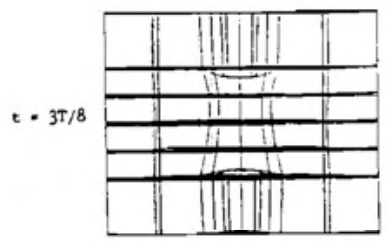

111

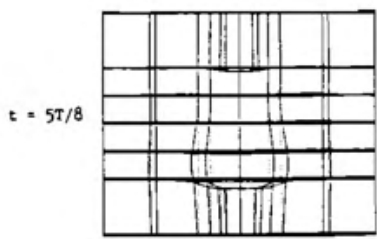

111

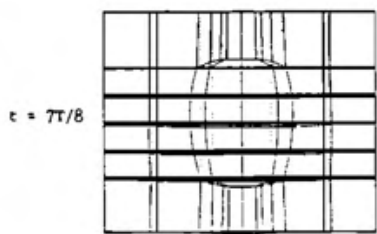

111

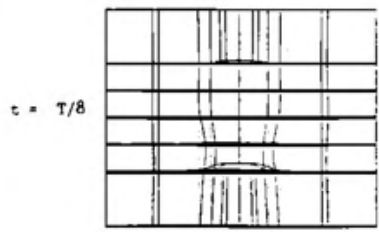

FIG. 15. Displacement field of the elementary cell of the Alberich anechoic coating, made of polyurethane, at $16 \mathrm{kHz}$, in the $x 0 z$ plane, at eight different moments, separated by one-eighth of the period.

ly attributed to the inaccuracies in the knowledge of the polyurethane properties.

The displacement field of the elementary cell is presented at $16 \mathrm{kHz}$, in the $x 0 z$ plane on Fig. 15 , as a cycle made up of eight displays. The amplitudes are again normalized to the maximum displacement amplitude. The resonance mode of the cavity at the minimum of the transmission coefficient is a combination of a radial motion of the hole wall and a small deformation of the cover layer. ${ }^{13,14}$ Nevertheless, the absorption mechanism is complex because the losses in the polyurethane introduce an important phase shift for the displacement across the thickness. Thus it seems that simple models can only provide a first insight in these cases and that a numerical modeling is required. Moreover, Fig. 15 shows that shear effects are large.

\section{Alberich anechoic coating made of silicone}

The second panel is made of silicone. Because the variations of the properties versus the frequency and the temperature are negligible, the properties are supposed to be constant in the frequency band of interest: $\rho=1000 \mathrm{~kg} / \mathrm{m}^{3}$, $E=1.8 \times 10^{6} \mathrm{~Pa}, v=0.49976$ and the loss angle for the Young's modulus is equal to $15 \%$. When the panel is excited 


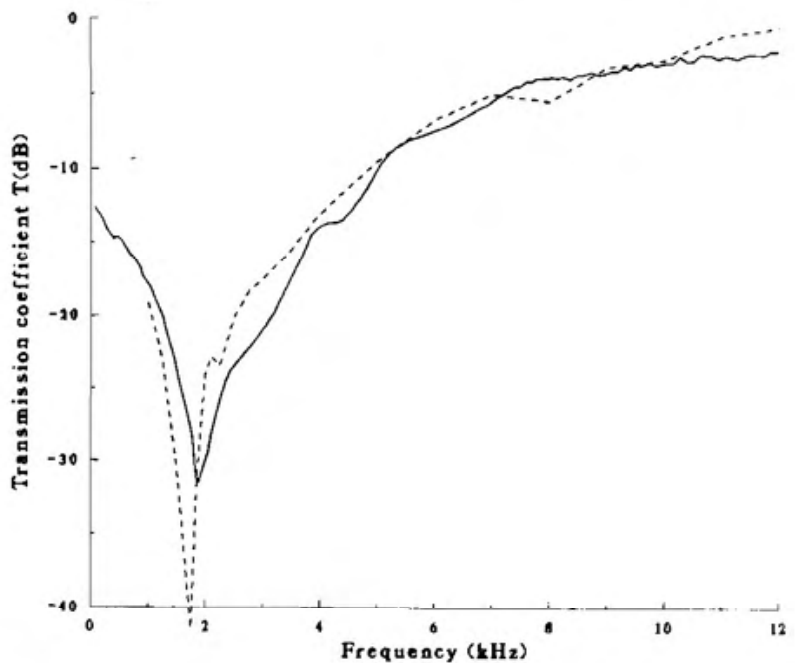

FIG. 16. Frequency variations of the transmission coefficient of the Alberich anechoic coating, made of silicone: full line: measurements; dashed line: FEM.

by a normal incident plane wave, the transmission coefficients obtained by the finite element method and by measurements are compared in Fig. 16. There is a good agreement between the two curves. The peak, at $1.75 \mathrm{kHz}$, is due to the excitation of a resonance mode of the cavity ${ }^{13,14}$ as well as in the case of the polyurethane Alberich anechoic coatings. But, the identification of the mode is difficult, because the losses in silicone, which are about $15 \%$, introduce again an important phase factor variation in the displacement field. Finally, it should be noted that mesh of Fig. 13 is also valid for the silicone panel because if the transverse velocity in silicone is 10 times smaller than transverse velocity in polyurethane, the frequency band of interest is also 10 times lower.

\section{CONCLUSION}

This paper has presented a finite element approach for modeling the acoustical behavior of doubly periodic structures. The results computed with the ATILA code have demonstrated that this approach is accurate and well suited to predict these behaviors. Good agreement has been found between the results obtained by the finite element model and by the measurements, or from other previously obtained theoretical models. Moreover, the efficiency and versatility of this finite element approach have been demonstrated. Indeed, the finite element method does not prescribe restrictive hypotheses to the displacement field, and it allows the modeling of any structure geometry by simply building the corresponding mesh, without any new development related to the method. Nevertheless, to obtain a nice agreement between finite element results and measurements, it has been pointed out that the properties of the material have to be known accurately. This knowledge is often difficult, because property values are varying rapidly versus temperature and frequency, in the range of interest. Moreover, it has also been pointed out that the mesh step selection must be done carefully. The $\lambda / 4$ criterion is sufficient for finite elements relying upon a quadratic interpolation but with respect to the smallest wave velocity. This velocity is classically the transverse wave velocity, but can be the flexural wave velocity in the cavity cover layers if these layers are sufficiently thin. Finally, computed displacement fields obtained for frequencies that are associated with large transmission losses allow a simple physical interpretation of the resonance effects.

Now, one of our intents is to extend this technique to the analysis of doubly periodic structures containing active composite materials, with a view to building optimized active gratings. Then, the modeling of triply periodic structures is projected. The modeling of the propagation of harmonic elastic waves through triply periodic structure can provide dispersion curves, giving results of physical interest: identification of propagation modes, cutoff frequencies, passband, stop band, ${ }^{33-36}$ as well as effective homogeneous properties of these structures and their validity limits.

\section{ACKNOWLEDGMENTS}

The authors wish to thank R. Bossut, from the Acoustics Department, Institut Supérieur d'Electronique du Nord, for his help in algorithm and program developments, and C. Audoly, from the Groupe d'Etude et de Recherche de Détection Sous-Marine (DCAN-Toulon) for his suggestions and for providing the measurements: This work was supported under contract by the Direction des Recherches, Etudes et Techniques (DRET, Paris).

\section{APPENDIX A: APPLICATION OF THE PERIODIC BOUNDARY CONDITIONS}

To take account of the phase relation (2) between nodes belonging to the $S 1$ and $S 3$ planes on the one hand, to the $S 2$ and $S 4$ planes on the other hand, the matrix equation ( 7 ) has to be modified. ${ }^{22,23,30}$ The corresponding relations are presented hereafter only in the case of an elastic cell (for the sake of simplicity), but they can be generalized to any cell, containing solid and fluid. The cell, the cross section of which, in the $x 0 y$ plane, is presented on Fig. Al, is divided into nine parts: surfaces $S 1, S 2, S 3$, and $S 4$, cornerlines $C 1$, $C 2, C 3$, and $C 4$ and inner domain In. The displacement vector $\mathbf{U}$ and the force vector $\mathbf{F}$ are then split into the corresponding nine parts. Due to relation (2), their components have to verify:

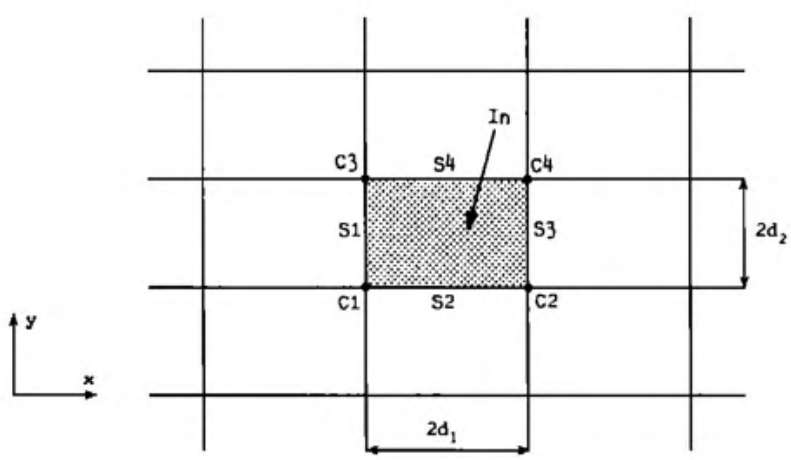

FIG. A1. Schematic representation of repetitive cells, in the $x 0 y$ plane 


$$
\begin{aligned}
& \left\{\mathbf{U}_{s 3}\right\}=e^{j \psi_{x}}\left\{\mathbf{U}_{s 1}\right\}, \quad\left\{\mathbf{U}_{s 4}\right\}=e^{j \psi_{y}}\left\{\mathbf{U}_{s 2}\right\}, \\
& \left\{\mathbf{U}_{c 2}\right\}=e^{j \psi_{x}}\left\{\mathbf{U}_{c 1}\right\}, \quad\left\{\mathbf{U}_{c 3}\right\}=e^{j \psi_{y}}\left\{\mathbf{U}_{c 1}\right\}, \\
& \left\{\mathbf{U}_{c 4}\right\}=e^{j\left(\psi_{x}+\psi_{y}\right)}\left\{\mathbf{U}_{C 1}\right\},
\end{aligned}
$$

where $\psi_{x}=2 d_{1} k \sin \theta \cos \varphi$ and $\psi_{y}=2 d_{2} k \sin \theta \sin \varphi$. Then, owing to the equilibrium of inter-connecting forces between two adjacent cells, relation (2) leads to analogous relations for the force vector. These equations are then substituted into the first line of Eq. (7). To do this, keeping in mind that only an elastic cell is considered here, the first line of Eq. (7) is rewritten under the form:

$$
\left[[K]-\omega^{2}[M]\right]\{\mathbf{U}\}=[Q]\{\mathbf{U}\}=\{\mathbf{F}\} .
$$

Using the same approach as in Refs. 22 and 23, the matrix $[Q]$ is partitioned following the nine parts. The resulting equation is

$$
\left[\begin{array}{llll}
R_{S 1, S 1} & R_{S 1, S 2} & R_{S 1, C 1} & R_{S 1,1 n} \\
R_{S 1, S 2}^{* T} & R_{S 2, S 2} & R_{S 2, C 1} & R_{S 2,1 n} \\
R_{S 1, C 1}^{* T} & R_{S 2, C 1}^{* T} & R_{C 1, C 1} & R_{C 1, \mathrm{In}} \\
R_{S 1, \mathrm{In}}^{* T} & R_{S 2, \mathrm{In}}^{* T} & R_{C 1, \mathrm{In}}^{* T} & R_{1 \mathrm{I}, \mathrm{In}}
\end{array}\right]\left[\begin{array}{c}
\mathrm{U}_{S 1} \\
\mathbf{U}_{S 2} \\
\mathbf{U}_{C 1} \\
\mathrm{U}_{\mathrm{In}}
\end{array}\right]=\{\mathbf{O}\},
$$

where

$$
\begin{aligned}
{\left[R_{S 1, S 1}\right]=} & {\left[Q_{S 1, S 1}+Q_{S 3, S 3}\right], } \\
{\left[R_{S 1, S 2}\right]=} & {\left[Q_{S 1, S 2}+Q_{S 1, S 4} e^{j \psi_{y}}+Q_{S 3, S 2} e^{-j \psi_{x}}\right.} \\
& \left.+Q_{S 3, S 4} e^{j\left(\psi_{y}-\psi_{x}\right)}\right], \\
{\left[R_{S 1, C 1}\right]=} & {\left[Q_{S 1, C 1}+Q_{S 3, C 2}+Q_{S 1, C 3} e^{j \psi_{y}}\right.} \\
& \left.+Q_{S 3, C 4} e^{j \psi_{y}}\right], \\
{\left[R_{S 1, \mathrm{In}}\right]=} & {\left[Q_{S 1, \mathrm{In}}+Q_{S 3, \mathrm{In}} e^{-\mu \psi_{x}}\right], } \\
{\left[R_{S 2, S 2}\right]=} & {\left[Q_{S 2, S 2}+Q_{S 4, S 4}\right], } \\
{\left[R_{S 2, C 1}\right]=} & {\left[Q_{S 2, C 1}+Q_{S 2, C 2} e^{j \psi_{x}}+Q_{S 4, C 3}\right.} \\
& \left.+Q_{S 4, C 4} e^{j \psi_{x}}\right], \\
{\left[R_{S 2, \mathrm{In}}\right]=} & {\left[Q_{S 2, \mathrm{In}}+Q_{S 4, \mathrm{In}} e^{-j \psi_{y}}\right], } \\
{\left[R_{C 1, C 1}\right]=} & {\left[Q_{C 1, C 1}+Q_{C 2, C 2}+Q_{C 3, C 3}+Q_{C 4, C 4}\right], } \\
{\left[R_{C 1, \mathrm{In}}\right]=} & {\left[Q_{C 1, \mathrm{In}}+Q_{C 2, \mathrm{In}} e^{-j \psi_{x}}+Q_{C 3, \mathrm{In}} e^{-j \psi_{y}}\right.} \\
& \left.+Q_{C 4, \mathrm{In}} e^{-j\left(\psi_{x}+\psi_{y}\right)}\right], \\
{\left[R_{\mathrm{In}, \mathrm{In}}\right]=} & {\left[Q_{\mathrm{In}, \mathrm{In}}\right], }
\end{aligned}
$$

where $\left[Q_{A, B}\right]$ is the notation for a submatrix which connects nodes belonging to subdomains $A$ and $B$ only. In terms of finite elements, this operation is the static condensation of degrees of freedom belonging to $S 3, S 4, C 2, C 3$, and $C 4$.

\section{APPENDIX B: APPLICATION OF THE PRESSURE AND PRESSURE NORMAL GRADIENT CONTINUITY EQUATIONS}

To take into account the effects of the external fluid domains, the pressure field in the finite element domain has to be matched with the fields given by the plane-wave expansions of Eqs. (3) and (5). To do this, the vector $\Phi$ is split into three parts, corresponding to surface $S_{+}$, inner domain $(I)$ and surface $S_{-}$. The formalism is the same for the two boundary surfaces, and the relations are only detailed in the case of surface $S_{+}$. Here, $\Phi_{+}$results from the merging of the elementary vectors

$$
\boldsymbol{\Phi}_{+}^{e}=\int_{S_{+}^{e}}\left\{N_{+}^{e}\right\}^{T} \Phi^{+} d S_{+}^{e},
$$

where $\Phi^{+}$is the pressure normal gradient on surface $S_{+}$ and $\left\{N_{+}^{e}\right\}^{T}$ is the vector of the shape functions associated with the bidimensional rectangular finite element $S_{+}{ }_{+}$, on surface $S_{+}$. Keeping only $\left(2 M_{x}+1\right) \cdot\left(2 M_{y}+1\right)$ terms in the series expansion (5), the parameters $M_{x}$ and $M_{y}$ depending on the mesh in directions $x$ and $y$ (Ref. 23), $\Phi^{+}$and $p^{+}$ are written in contracted notation:

$$
\begin{aligned}
& \Phi^{+}=(\underbrace{j\left(\alpha_{n} x+\beta_{m} y\right)})^{T}(\underbrace{T_{n m}^{\Phi} \mathrm{e}^{j k_{n m^{2}}}}), \\
& p^{+}=\left(\mathrm{e}^{j\left(\alpha_{n} x+\beta_{m} y\right)}\right)^{T}\left(T_{n m}^{p} \mathrm{e}^{j k_{n m}{ }^{2}}\right) \text {. }
\end{aligned}
$$

Then, the relation between the pressure $p^{+}$and its normal derivative $\Phi^{+}$provides the equation

$$
T_{n m}^{\Phi} e^{j k_{n m^{2}}}=n_{i} \frac{\partial\left(T_{n m}^{p} e^{j k_{n m^{2}}}\right)}{\partial x_{i}}=j k_{n m}\left(T_{n m}^{p} e^{j k_{n m^{2}}}\right),
$$

which becomes, in matrix form:

$$
(\underbrace{\Phi}_{n m} \mathrm{e}^{j k_{n m^{2}}})=\left[D^{+}\right](\overbrace{}^{T_{n m}^{p} \mathrm{e}^{k_{n m^{2}}}}) .
$$

Here, the matrix $\left[D^{+}\right]$is a diagonal matrix of order $\left(2 M_{x}+1\right) \cdot\left(2 M_{y}+1\right)$ and of generating term $j k_{n m}$. Taking into account Eq. (B4) in the expression of $\Phi_{+}^{e}$ provides

$$
\begin{aligned}
\Phi_{+}^{e}= & \int_{S_{+}^{e}}\left\{N_{+}^{e}\right\}^{T}\left(\mathrm{e}^{j\left(\alpha_{n} x+\beta_{m} y\right)}\right)^{T}\left[D^{+}\right] \\
& \times\left(T_{n m}^{p} e^{j k_{n m^{z}}}\right) d S_{+}^{e} .
\end{aligned}
$$

Using a Fourier series expansion of the pressure, $p^{+}$can be again expressed as a product of two vectors of order $\left(2 M_{x}+1\right) \cdot\left(2 M_{y}+1\right):$

$$
\begin{aligned}
& p^{+}=\sum_{n=-M_{x} m-}^{+M_{x}} \sum_{-M_{y}}^{+M_{y}} c_{n m} e^{j\left(\alpha_{u} x+\beta_{m} y\right)}, \\
& p^{+}=\left(\mathrm{e}^{j\left(\alpha_{n} x+\beta_{m} y\right)}\right)^{T} \mathbf{C},
\end{aligned}
$$

where vector $\mathbf{C}$ contains terms $c_{n m}$ :

$$
\begin{aligned}
c_{n m}= & \frac{1}{2 d_{1}} \frac{1}{2 d_{2}} \int_{-d_{1}}^{+d_{1}} \int_{-d_{2}}^{+d_{2}} p^{+}(x, y) \\
& \times e^{-j\left(\alpha_{n} x+\beta_{m} y\right)} d x d y .
\end{aligned}
$$

The pressure $p^{+}(x, y)$ can be related to the pressure nodal values on surface $S_{+}$, with the help of shape functions. Then

$$
\begin{aligned}
c_{n m}= & \left(\sum_{e} \frac{1}{2 d_{1}} \frac{1}{2 d_{2}} \int_{S_{+}^{e_{+}}} e^{-j\left(\alpha_{,} x+\beta_{m} y\right)}\right. \\
& \left.\times\left\{N_{+}^{e}\right\}^{T} d x d y\right) \mathbf{P}_{+}=\left\{A_{n m}^{+}\right\}^{T} \mathbf{P}_{+},
\end{aligned}
$$

where $\left\{A_{n m}^{+}\right\}^{T}$ is a line matrix. Finally, the vector $\mathbf{C}$ can be written under the following form:

$$
\mathbf{C}=\left[A^{+}\right] \mathbf{P}_{+} .
$$

The matrix $\left[A^{+}\right]$contains the $\left(2 M_{x}+1\right) \cdot\left(2 M_{y}+1\right)$ 
lines of Eq. (B8) and its number of columns is the number of nodes on the surface $S_{+}$. Then, the coefficients $T_{n m}^{p}$ are easily known with the help of Eq. (B2), (B6), and (B9):

$$
\left(T_{n m}^{p} \mathrm{e}^{j k_{n m}^{2}}\right)=\mathbf{C}=\left[A^{+}\right] \mathbf{P}_{+} \cdot
$$

The vector $\Phi_{+}^{e}$ from Eq. (B5) is written as

$$
\begin{aligned}
\Phi_{+}^{e}= & \left(\int_{S_{+}^{e}}\left\{N_{+}^{e}\right\}^{T}\left(e^{j\left(\alpha_{m} x+\beta k_{m} y\right)}\right)^{T}\right. \\
& \left.\times\left[D^{+}\right]\left[A^{+}\right] d S_{+}^{e}\right) \mathbf{P}_{+} .
\end{aligned}
$$

Merging elementary vectors $\boldsymbol{\Phi}_{+}{ }_{+}$, the nodal values of the pressure normal gradient $\boldsymbol{\Phi}_{+}$on the fluid surface $S_{+}$is

$$
\begin{aligned}
\boldsymbol{\Phi}_{+}= & {\left[\sum_{e} \int_{S_{+}^{e}}\left\{N_{+}^{e}\right\}^{T}(\overbrace{e^{\left(\alpha_{n} x+\beta k_{m} y\right)}})^{T}\right.} \\
& \left.\times\left[D^{+}\right]\left[A^{+}\right] d S_{+}^{e}\right] \mathbf{P}_{+} \\
\boldsymbol{\Phi}_{+}= & 2 d_{1} 2 d_{2}\left[A^{+}\right]^{\bullet T}\left[D^{+}\right]\left[A^{+}\right] \mathbf{P}_{+} \\
= & {\left[\Delta^{+}\right] \mathbf{P}_{+} . }
\end{aligned}
$$

The same processing on the surface $S_{-}$gives the $\left[\Delta^{-}\right]$ matrix. But for this surface, the nodal vector of the pressure and its normal derivative are written as a sum of the incident and the reflected vectors

$$
\boldsymbol{\Phi}_{-}=2 \boldsymbol{\Phi}_{i}+\left[\Delta^{-}\right] \mathbf{P}_{-},
$$

where the vector $\Phi_{i}$ contains the nodal values of the incident pressure normal gradient. Finally, the matrices $\left[\Delta^{+}\right]$and $\left[\Delta^{-}\right]$are incorporated in system (7).

'E. Burke and V. Twersky, "On scattering of waves by an infinite grating of elliptic cylinders," IEEE Trans. Antennas Propag. AP-14, 465-480 (1966).

${ }^{2} \mathrm{G}$. Dumery, "Sur la diffraction des ondes sonores par des grilles ou des réseaux d'obstacles," Acustica 18, 334-341 (1967).

${ }^{3}$ V. Vovk, V. T. Grinchenko, and L. A. Kononuchenko, "Diffraction of a sound wave by a plane grating formed by hollow elastic bars," Akust. Zh. 22, 201-205 (1976) [Sov. Phys. Acoust. 22, 113-115 (1976)].

${ }^{4}$ G. A. Brigham, J. J. Libuha, and R. P. Radlinski, "Analysis of scattering from large planar gratings of compliant cylindrical shells," J. Acoust. Soc. Am. 61, 48-59 (1977).

${ }^{5}$ R. P. Radlinski and M. M. Simon, "Scattering by multiple gratings of compliant tubes," J. Acoust. Soc. Am. 72, 607-614 (1982).

${ }^{\circ} \mathrm{C}$. Audoly, "Diffraction d'une onde plane par un réseau de tubes compliants," Acustica 64, 159-163 (1987).

'R. P. Radlinski, "Scattering from multiple gratings of compliant tubes in a viscoelastic layer," J. Acoust. Soc. Am. 85, 2301-2310 (1989).

${ }^{8} \mathrm{C}$. Audoly and G. Dumery, "Etude d'écrans sous-marins constitués de tubes élastiques," Acustica 69, 263-269 (1989).

${ }^{9} \mathrm{C}$. Audoly, "Modeling of compliant tube underwater reflectors," J. Acoust. Soc. Am. 87, 1841-1847 (1990).

${ }^{10} \mathrm{C}$. W. Kosten and C. S. Zwikker, Sound Absorbing Materials (Elsevier, New York, 1949).

"H. Oberst, Resonant Sound Absorbers, Technical Aspects of Sound, edited by E. G. Richardson (Elsevier, Amsterdam, 1957), Vol. II.

${ }^{12} \mathrm{G}$. C. Gaunaurd, "One-dimensional model for acoustic absorption in a viscoelastic medium containing short cylindrical cavities," J. Acoust. Soc. Am. 62, 298-307 (1977).
${ }^{13}$ R. Lane, “Absorption mechanisms for waterborne sound in Alberich anechoic layers," Ultrasonics 28-30 (January, 1981).

${ }^{14} \mathrm{G}$. C. Gaunaurd, "Comments on "Absorption mechanisms for waterborne sound in Alberich anechoic layers,' " Ultrasonics 90-91 (March, 1985).

${ }^{15} \mathrm{G}$. C. Gaunaurd and H. Überall, "Resonance theory of the effective properties of perforated solids," J. Acoust. Soc. Am. 71, 282-295 (1982); Errata, J. Acoust. Soc. Am. 72, 371 (1982).

${ }^{16} \mathrm{G}$. C. Gaunaurd, "Resonance effects of the ultrasonic effective properties of particulate composites," J. Acoust. Soc. Am. 74, 305-313 (1983).

"J. D. Achenbach, Y. C. Lu, and M. Kitahara, "3-D reflection and transmission of sound by an array of rods," J. Sound Vib. 125, 463-476 (1988).

${ }^{18} \mathrm{P}$. Vidoret, "Reflection and transmission characteristics of an Alberich type acoustic barrier," Proc. Symposium on Physical Acoustics, 19-22 June 1990 (Kortrijk, Belgique, 1990), pp. 657-664.

${ }^{19}$ A. Lakhtakia, V. V. Varadan, and V. K. Varadan, "Reflection characteristics of an elastic slab containing a periodic array of elastic cylinders: $\mathrm{SH}$ wave analysis," J. Acoust. Soc. Am. 80, 311-316 (1986).

${ }^{20}$ A. Lakhtakia, V. V. Varadan, and V. K. Varadan, "Reflection characteristics of an elastic slab containing a periodic array of circular elastic cylinders: $P$ and $S V$ wave analysis," J. Acoust. Soc. Am. 83, 1267-1275 (1988).

${ }^{21}$ A. J. Kalinowski, "Steady state solutions to the dynamically loaded periodic structures," NASA Conference Publication 2131, Eighth NASTRAN User's Colloquium (1979).

${ }^{22}$ A. C. Hennion, R. Bossut, J. N. Decarpigny, and C. Audoly, “Analysis of the scattering of a plane acoustic wave by a periodic elastic structure using the finite element method: application to compliant tube gratings," J. Acoust. Soc. Am. 87, 1861-1870 (1990).

${ }^{23}$ A. C. Hladky-Hennion, "Application de la méthode des éléments finis à la modélisation de structures périodiques utilisées en acoustique," Thesis, Université des Sciences et Techniques de Lille (1990).

${ }^{24}$ T. C. Ma, R. A. Scott, and W. H. Yang, "Harmonic wave propagation in an infinite elastic medium with a periodic array of cylindrical pores," J. Sound Vib. 71, 473-482 (1980).

${ }^{25} \mathrm{~J}$. N. Decarpigny, “Application de la méthode des éléments finis à l'étude de transducteurs piézoélectriques," Thesis, Université des Sciences et Techniques de Lille (1984).

${ }^{26}$ J. N. Decarpigny, J. C. Debus, P. Tierce, B. Tocquet, and D. Boucher, "In-air analysis of piezoelectric Tonpilz transducers in a wide frequency band using a mixed finite element-plane wave method," J. Acoust. Soc. Am. 78, 1499-1507 (1985).

${ }^{27} \mathrm{R}$. Bossut and J. N. Decarpigny, "Finite element modeling of radiating structures using dipolar damping elements," J. Acoust. Soc. Am. 86, 1234-1244 (1990)

${ }^{28}$ B. Hamonic, J. C. Debus, J. N. Decarpigny, D. Boucher, and B. Tocquet, "Analysis of radiating thin shell sonar transducers using the finite element method," J. Acoust. Soc. Am. 86, 1245-1253 (1990).

${ }^{29}$ B. Dubus, J. C. Debus, J. N. Decarpigny, and D. Boucher, “Analysis of mechanical limitations of high power piezoelectric transducers using finite element modeling," Ultrasonics 29, 201-207 (1991).

${ }^{30} \mathrm{D}$. J. Mead, "A general theory of harmonic wave propagation in linear periodic systems with multiple coupling," J. Sound Vib. 27, 235-260 (1973).

${ }^{31}$ The $\lambda / 4$ criterion is related to the 20 - and 15 -node isoparametric finite elements used in this work and due to the fact that the shape function variations are quadratic with respect to the coordinates along an element side. See, for example, Refs. 25-28 above.

${ }^{32} \mathrm{O}$. B. Wilson, An Introduction to the Theory and Design of Sonar Transducers (Peninsula, Los Altos, 1988).

${ }^{33}$ S. Baste and A. Gerard, "Etude des modes guidés dans les composites tridimensionnels," Rev. Acoust. 78, 43-48 (1986).

${ }^{34} \mathrm{D}$. Bai and J. B. Keller, "Sound waves in a periodic medium containing rigid spheres," J. Acoust. Soc. Am. 82, 1436-1441 (1987).

${ }^{35} \mathrm{~J}$. D. Achenbach and M. Kitahara, "Harmonic waves in a solid with a periodic distribution of spherical cavities," J. Acoust. Soc. Am. 81, 595598 (1987).

${ }^{36}$ M. Yamaguchi, K. Y. Hashimoto, and H. Makita, “Finite element method analysis of dispersion characteristics for 1-3 type piezoelectric composites," 1987 IEEE Ultrason. Symp. Proc. 657-661 (1987). 